Introduction: One way to examine the extent to which the stress associated with a breast cancer experience (BC) impacts stress-related physiological mechanisms is to study the secretion patterns of associated biomarkers. Unlike cortisol and $\alpha$-amylase (SAA), biomarkers of immune functioning such as secretory immunoglobulin A (SIgA) have rarely been examined in $B C$ survivors.

Aim of the study: This study had two principal aims: the first was to evaluate the basal secretion profiles of SIgA as well as its response to an acute stressor as a marker of immune health in BC survivors and women with no history of $\mathrm{BC}$, and the second was to determine how SIgA stress-related patterns compare to published cortisol and SAA patterns in the same women.

Results: Overall, the findings indicate that $\mathrm{BC}$ survivors exhibit a blunted cortisol reaction to an acute stressor, a generally elevated diurnal SAA concentration pattern, and normal SIgA profiles, compared to women with no history of cancer. This study serves as a foundation for future research to elucidate the relationships between $\mathrm{BC}$ experience variables, stress biomarkers, and health outcomes in BC survivors.

Key words: cortisol, $\alpha$-amylase, secretory immunoglobulin A, breast cancer survivorship, stress, immunity.

Contemp Oncol (Pozn) 2018; 22 (3): 191-201 DOI: https://doi.org/10.5114/wo.2018.78946

\section{Salivary secretory immunoglobulin A reactivity: a comparison to cortisol and $\alpha$-amylase patterns in the same breast cancer survivors}

Maude Lambert, Marie-Ėve Couture-Lalande, Kelly Brennan, Aldin Basic, Sophie Lebel, Catherine Bielajew

University of Ottawa, Ottawa, Canada

Breast cancer survivors experience several physiological sequelae following a cancer experience, even several years after the end of treatment
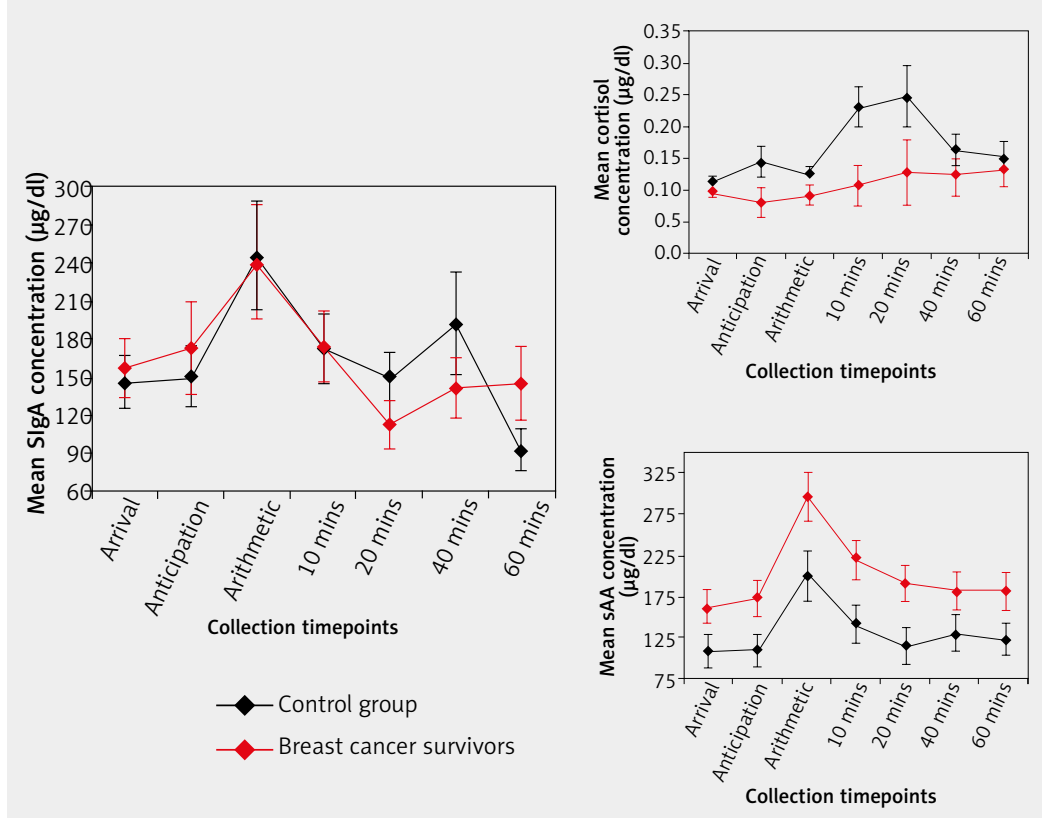

\section{Introduction}

Cancer is the cause of nearly $30 \%$ of all deaths in Canada and is quickly becoming a worldwide pandemic [1]. In men, prostate cancer is the leading cancer site, while in women, it is breast cancer (BC), with a lifetime risk of developing it estimated to be one in nine women [1]. The mortality rates of BC in Canada are now the lowest since 1950, with a five-year survival rate of $88 \%$ [1]. Continued advances in BC detection and treatment strategies along with the aging and growth of the population have resulted in an ever-increasing number of survivors.

Despite general agreement that a cancer diagnosis generates significant stress due to difficult treatment procedures, drastic changes in daily routine, and family and work concerns [2], what is less obvious is that the cancer experience continues well beyond the end of treatment. In fact, based on several cross-sectional and longitudinal studies, the symptom burden linked to cancer survivorship can persist for more than 10 years following treatment [3]. Unrelenting symptoms can include fatigue, pain, distress, and cognitive impairment [4]. 
It has long been known that chronic stress can severely alter human biological systems in many ways [5]. The stress associated with the cancer trajectory - from diagnosis to post-treatment - has been shown to suppress or dysregulate innate and adaptive physiological responses [6]. One way to examine the extent to which stress impacts stress-related physiological mechanisms in BC survivors is to evaluate the secretion patterns of associated biomarkers. Several biomarkers are considered to be very reliable and sensitive indicators of an individual's biological response (biochemical, physiological, cellular, or behavioural responses) to different types of stressors [7].

\section{Salivary cortisol as a stress biomarker}

The cancer experience has been shown to cause a dysregulation of the hypothalamic-pituitary-adrenal (HPA) axis, a system that tightly regulates stress responses [8]. Studies have found associations between HPA dysregulation and mortality and disease severity in BC patients [9]. Cortisol, which is a steroid hormone secreted in response to stress, is often used to assess the activity of the HPA system and has received extensive attention in research on stress physiology. Situations characterised as unpredictable, uncontrollable, and threatening (such as a BC diagnosis) elicit increases in cortisol concentrations [10].

A diurnal cortisol pattern that peaks at awakening and gradually decreases throughout the day is the typical response of a functional HPA axis [11]. Flatter diurnal cortisol patterns, or concentration patterns that remain fairly unchanged throughout the day, occur significantly more often in BC survivors than in healthy individuals [8, 12]. During acute stress situations, cortisol secretion is temporarily increased and concentration levels usually return to normal within one or two hours after stress cessation [13]. While cortisol is essential for regulating bodily functions and responding to environmental challenges [10], sustained high cortisol levels can have damaging effects on the human body and have been associated with depression [14], obesity [15], diabetes [16], and social isolation [17]. Low levels of cortisol, on the other hand, have been linked to pain, fatigue, high stress sensitivity [18], and stress-related disorders such as post-traumatic stress disorder and fibromyalgia syndrome [19].

In our laboratory, we have examined the salivary cortisol diurnal rhythmicity and reactivity in long-term BC survivors compared to women with no history of BC [20]. Although we found no group differences in their diurnal cortisol secretion (Fig. 1B), the groups showed significant distinctive patterns in response to an acute stressor: the concentrations of cortisol in women with no history of cancer peaked at 10-20 minutes following the stressor whereas the levels in $\mathrm{BC}$ survivors remained fairly blunted over the same time course (see Fig. 2B). While our study revealed that both groups appraised psychological stress in an almost identical fashion, survivors demonstrated a considerably diminished physiological response to the acute stressor. Similar findings have also been reported from other laboratories [8, 21, 22].

\section{Salivary $\alpha$-amylase as a stress biomarker}

More recently, investigators have been studying salivary $\alpha$-amylase (SAA), an indicator of sympathetic nervous system (SNS) activity, for its potential as a stress biomarker. To date, a number of studies have suggested that sAA responds to both physical and psychological stress [23, 24]. Its concentrations have been found to be directly related to the subjective stress levels of BC survivors [25]. Distinctly different from that of cortisol, typical sAA concentrations are reduced significantly within 60 minutes after awakening and gradually increase throughout the day, reaching their peak in the late afternoon or evening $[24,26]$.

Salivary $\alpha$-amylase levels have also been measured in response to different acute stressors such as writing a test [27] and skydiving [28]. Both studies revealed significant increases in SAA levels the days preceding the expected stressor. Other studies reported that sAA activity was significantly higher in samples collected after subjects were exposed to an acute stressor compared to samples collected at any other time [29]. Furthermore, the total sAA released after exposure to an acute stress has been found to be positively correlated with heart rate, pain intensity, and the total amount of cortisol released [30, 31]. This suggests the coordination between the HPA system and the SNS in their response to acute stress.

Based upon the results of our previous study and findings from other groups of researchers, we reasoned that HPA regulation abnormalities in BC survivors, as interpreted from cortisol patterns, would probably be accompanied by SNS dysregulation [32]. Consequently, we explored the circadian and reactive profiles of SAA in the same groups of participants who showed blunted cortisol patterns in response to acute stress [20]. We found that, while BC survivors had significantly higher diurnal and reactive SAA patterns than women with no history of cancer, the overall pattern was the same in both groups (Fig. 1C, Fig. 2C). In other words, the SAA difference in acute stress responses was not related to a specific acute stress response but was instead reflected by a heightened basal level of concentration.

\section{Salivary secretory immunoglobulin A as a stress and immune biomarker}

One of the most important lines of defence against human pathogens is the mucosal immune system [33]. The main immunoglobulin, secretory A (SIgA), is the predominant effector of that specific system and has often been used as a marker of immune competence [33, 34]. Immunoglobulins are antibodies secreted by plasma cells that combine with antigens and direct an immune response against them [35]. Secretory immunoglobulin $A$ is a component of the adaptive immune system and works alongside other innate mucosal defence factors such as $\alpha$-amylase, lactoferrin, and lysozyme in order to fight surfacing pathogens [33, 36, 37]. Similar to cortisol, SIgA concentration peaks in the morning and then progressively declines to its nadir by the evening [38]. Several other studies have replicated those findings [31, 39, 40]. 
Components of saliva, including SIgA, are crucial indicators of health. Low levels of salivary SIgA can signal bacterial and viral infections in an individual [41-43]. For instance, patients diagnosed with Acquired Immune Deficiency Syndrome are found to have a significantly lower level of salivary SIgA, resulting in very frequent oral infections [44]. Moreover, an earlier study by Brown et al. [45] found that cancer recurrence was associated with elevated SIgA levels, which may indicate a specific secretory response to tumour. They suggested that SIgA could potentially be used to distinguish patients who are at risk of recurrence.

\section{Chronic stress and secretory immunoglobulin A}

The relationship between stress, immunity, and environmental influences has long been of interest to researchers who investigate the effects of stress on health [43]. There is widespread agreement that psychological stress increases susceptibility to disease [46-48]. The duration of the stressor seems to play an important role in the immune response to psychological stress [49]. Several studies have reported that chronic or long-term stress has a suppressive effect on immune functioning and SIgA $[6,50]$. For instance, a diminished SIgA level has been found in continually neglected toddlers [51], soldiers [52], and nurses enduring chronic work stress [53]. While this suggests that a long-term cancer experience might suppress immune function, most research on stress responses in BC survivors has been focused on more traditional stress-related biomarkers such as cortisol and sAA.

\section{Acute stress and secretory immunoglobulin A}

Very few studies have explored the effects of acute stress on immune system responses. However, the few that have claim an association between acute stress and an increase in immune functioning and SIgA levels [6, 36]. This activation in response to stress highlights the immune system's fundamental ability to protect the body from disease [49]. Elevated SIgA levels were found in soccer coaches during a crucial game [54] and in police officers during a grave accident [55]. Laboratory-induced stress, such as speech and arithmetic tasks, also supports this claim $[37,56]$.

\section{The present study}

To our knowledge, no study to date has compared the profiles of several stress biomarkers in the same cancer survivors. Because cortisol and SAA are secreted by different hormonal systems and have unique diurnal secretion patterns [26,57], the measurement of both biomarkers in tandem following stress induction provides a more comprehensive understanding of different aspects of stress regulation and its mechanisms. Moreover, the significant gap in the literature on SIgA diurnal patterns of concentrations and its response to an acute stressor highlights the need for more research on this under-explored biomarker. The examination of SIgA concentrations in BC survivors not only gives us more information on the activity of their stress systems but also provides a mean of examining their immune function.
To that end, the aim of this current study was to investigate the relationship between SIgA reactivity and the stress biomarkers, cortisol and SAA, in the same individuals; the latter findings have been published [20, 32]. The first objective was to examine the basal secretion profiles of SIgA as well as its response to an acute stressor in BC survivors, and to compare these to women with no history of BC. The second objective was to determine the association between SIgA diurnal and reactive concentration patterns in $B C$ survivors and how these relate to their cortisol and SAA patterns. We hypothesised the following: First, that the stress associated with a BC experience would result in abnormalities in SIgA diurnal concentration patterns. Second, based on previous literature highlighting the link between the HPA axis and immunocompetence [38, 58], we further hypothesised that BC survivors and women with no history of cancer would exhibit differences in their SIgA response to the Trier Social Stress Test (TSST), a widely-used stress-inducing laboratory protocol, (see Methods section for further description). Third, we also examined whether medical factors related to $B C$ such as cancer stage, time since diagnosis, and treatment regimen had any impact on the SIgA response observed in survivors.

\section{Material and methods}

\section{Participants}

Via printed advertisements and cancer support groups, a total of 48 women were recruited: 22 women BC survivors and 26 women with no history of cancer. Table 1 characterises the participants by age, ethnicity, level of education, and family income. The medical characteristics of the BC survivors are shown in Table 2. Please note that Table 1 and Table 2 are also reported in [20] and in [32], as they depict the same group of individuals.

The eligibility inclusion criteria for BC survivors included: a) a diagnosis of $B C$ at least one year prior, b) completion/cessation of all cancer-related treatments at least six months earlier, and c) the ability to provide informed consent. Individuals with: a) history of other cancers (except non-invasive skin cancer and cervical cancer), b) substance abuse problems, or c) any major disabling conditions interfering with their quality of life (for example, psychiatric disorders) were excluded from the present study. Women who were breast feeding, pregnant, taking any medication that could alter hormonal secretion (e.g. hydrocortisone, hypnotics, benzodiazepines), or had bleeding gums were also excluded. The women in the control arm had to meet the following criteria: a) completion of routine mammography screening with negative results, b) no history of cancer, as well as the exclusion criteria listed in c) above.

Prior to inclusion in the study, written informed consent was obtained from all participants. Participants received $\$ 50$ as travel compensation and a chance to win a \$250 prize. The study was approved by the University of [location withheld for blinded review] Ethics Review Board and was conducted at the [location withheld for blinded review] Laboratory. 


\section{Measures of stress}

\section{Salivary secretory immunoglobulin A}

Extraction of SIgA from saliva was carried out using a commercially available highly-sensitive enzyme-linked immunosorbent assay (ELISA). The assay kits and the protocol were obtained from Salimetrics, State College, PA, USA [59].

\section{Trier Social Stress Test}

The Trier Social Stress Test (TSST) has become the gold standard for evaluating acute stress response in the laboratory setting $[60,61]$. Its effects on biomarker concentration patterns, including cortisol, sAA, and SIgA, have been shown in past research $[56,62,63]$. The TSST protocol we used had two major components: 1) a mock interview during which participants had to give a five-minute free speech to a panel of three confederate evaluators acting as a hiring committee, and 2) a five-minute arithmetic task which required that the participants count down from 1022 by increments of 13 as quickly as possible [64].

\section{Visual Analog Scale}

The Visual Analog Scale (VAS) is a bipolar line quantifying a specific characteristic across a continuum [65]. In this context, it was used to measure participants' subjective stress response before, during, and after the TSST. Participants were asked to mark a spot on the line that indicated their subjective stress appraisal. Based on the statement "I feel stressed", participants estimated their perceived stress between 0 (not at all) to 100 (very much). Their score was based on the distance between the left end of the line and the appraisal mark.

\section{Questionnaires}

Participants were asked to complete a series of questionnaires in order to assess their socio-demographic characteristics and stress perception. The package included: a) socio-demographic questionnaire requesting information about participants' general life history, health history (for BC survivors, this included questions related to their BC history), and life habits; b) the Daily Stress Inventory, a 58-item questionnaire inquiring about recent (past $24 \mathrm{~h}$ ) stressful events and their intensity [66]; c) the Perceived Stress Scale of 14 items measuring subjective appraisals of stressful situations in the past month [67]; and d) the Life Experiences Survey, a questionnaire used to record the frequency and impact of life events, both positive and negative, that occurred in the past year [68].

\section{Procedure}

Eligible participants were asked to attend two laboratory visits at the University of [location withheld for blinded review]. In order to promote neutrality in the results and also to control for potential confounding variables such as practice and expectation effects, participants were only told that the aim of the study was to examine the effect of stress. The other details of the experiment remained unknown to them.
The first meeting, which lasted about 30 minutes, served to obtain informed consent and to teach participants the correct method of collecting saliva samples at home for the purpose of the diurnal analyses. They were asked to: 1) rinse their mouth 10 minutes before collection (as a way to avoid sample dilution), 2) place the salivette directly under their tongue for a full three minutes, 3) avoid touching the salivette with their fingers, and 4) store the salivettes in the supplied containers in the refrigerator until delivery to the lab. We also asked participants to avoid smoking and drinking alcohol for $24 \mathrm{~h}$ before sample collection and ingesting caffeine products and exercising for an hour prior to collection. Participants were then given a home kit of pre-labelled salivettes. They were required to collect a total of five saliva samples at home on each of two consecutive days at the following times: upon waking, $30 \mathrm{~min}$ after waking, at $1200 \mathrm{~h}, 1600 \mathrm{~h}$, and $2100 \mathrm{~h}$. In the event that participants did not have access to a refrigerator to store their salivettes until delivery to the laboratory, we provided them with an insulated lunch bag and ice packs.

The second laboratory visit was the stress induction portion of the study (the TSST), which lasted about two hours; it was scheduled within seven days following the first visit. In the laboratory, seven saliva samples were collected. Figure 3 is a schematic representation of the procedure of the laboratory session. The first one, labelled "arrival", was retrieved as soon as the participant entered the laboratory. They were then taken into the testing room where a mock panel of committee members explained the TSST instructions. The first task of the TSST involved the preparation and delivery of a five-minute speech about the reasons why they believe they are the most suitable candidate for the mock job position. The second task, the arithmetic task, followed immediately after. The saliva sample collected between the speech preparation and the speech delivery was labelled "anticipation" and the one upon completion of the arithmetic task, "arithmetic". At each time-point that a saliva sample was collected, participants had to rate their subjective stress level on the VAS. Upon completion of the TSST, participants were asked to relax in a room for one hour and complete a series of questionnaires measuring their perceived stress, anxiety, and fear of recurrence. Four additional saliva samples were collected at 10, 20, 40, and 60 minutes during this phase. Finally, participants were debriefed and the true aim of the study was explained to them. Each saliva sample was divided into three aliquots immediately after collection and then transferred to separate Eppendorf tubes, in order to avoid multiple freezing and thawing cycles. To foster optimal stability of the samples, they were stored at $-80^{\circ} \mathrm{C}$ until processed [69]. Cortisol was analysed first, followed by SAA, and finally SIgA.

\section{Results}

\section{Participants characteristics}

Participant demographics and characteristics are depicted in Table 1 (also depicted in [20] and [32]). In short, the study had a total of 48 participants, which included $22 \mathrm{BC}$ survivors and 26 women with no history of BC. Both 
Table 1. Demographic characteristics of participants

\begin{tabular}{lcc} 
Demographic characteristics & \multicolumn{2}{c}{ Participants } \\
\cline { 2 - 3 } Age (years): mean [SD] (range) & Breast cancer survivors $(n=22)$ & Healthy women $(n=26)$ \\
Ethnicity & $58.9[10.1](39-81)$ & $57.4[11](41-73)$ \\
White & No. of participants (\%) & No. of participants (\%) \\
Black & $20(90.9)$ & $23(88.5)$ \\
Asian & - & $1(3.8)$ \\
First nations & - & $2(7.7)$ \\
Highest level of education & $2(9.1)$ & - \\
High school & & \\
College & $6(27.3)$ & $9(34.6)$ \\
Bachelor's degree & $4(18.2)$ & $4(15.4)$ \\
Graduate degree & $11(50.0)$ & $7(26.9)$ \\
Family income (CDN) & $1(4.5)$ & $6(23.0)$ \\
Under $\$ 40,000$ & & \\
\$40,000 to $\$ 79,999$ & $3(15)$ & $5(20.8)$ \\
\$80,000 to $\$ 119,999$ & $10(50)$ & $10(41.7)$ \\
\$120,000 and over & $5(25)$ & $5(20.8)$ \\
* & $2(10)$ & $4(16.7)$
\end{tabular}

* breast cancer survivor group $(n=20)$, control group $(n=24)$

groups had an average age in the late 50s (t-test group difference $p=0.488$; note that $\mathrm{BC}$ survivors tend to be older women and thus the age range of our sample would be truncated by definition), a similar number of women with postmenopausal status ( $\chi^{2}$ group difference $p=0.147$ ), and about $90 \%$ of each group self-identified as White. To our knowledge, aside from a few cases of hypertension, diabetes, and osteoarthritis, no serious or untreated medical conditions were present in participants. The medical characteristics of the BC survivors are shown in Table 2 (also see [20] and [32]). Their mean age at diagnosis was $54 \pm 9$ years (SD), and they were all recruited about five years after their diagnosis. The majority of the participants identified with stage $1 \mathrm{BC}$. Almost half of the participants underwent a lumpectomy procedure. All participants went through chemotherapy, radiation, hormone therapy, and/ or surgery; most received a combination of these treatments. Two participants suffered a recurrence of $B C$ and were therefore excluded from the study.

\section{Data analysis}

We eliminated from our data analysis participants who had more than two missing saliva samples (out of 10) for their diurnal data and more than three missing samples (out of seven) for their reactive data. Based on those criteria, four participants associated with the diurnal data and 10 associated with the reactive data had to be excluded. Diurnal analyses were therefore performed on 44 participants, and reactive analyses were performed on 38 participants. The single missing value was replaced using the EM algorithm in SPSS. Standard data and cleaning procedures were applied to the data before analysis including tests of normality and skewness; no transformations were required. All analyses were conducted using SPSS (v23).

A series of mixed-design analysis of variance (ANOVA) were conducted to determine group differences in SIgA
Table 2. Medical characteristics of the breast cancer survivors

$\begin{array}{lc}\text { Medical characteristics } & \begin{array}{c}\text { Breast cancer } \\ \text { survivors }(\boldsymbol{n}=\mathbf{2 2})\end{array} \\ \text { Mean age (years) of diagnosis (SD) } & 54.1(8.7) \\ \text { Mean time (years) since diagnosis (SD) } & 4.6(3) \\ \text { Stage of breast cancer } & \text { No. of participants (\%) } \\ 0 & 4(18.2) \\ 1 & 10(45.5) \\ 2 & 5(22.7) \\ 3 & 3(13.6) \\ \text { Type of surgery } & \\ \text { Unilateral mastectomy } & 6(27.3) \\ \text { Bilateral mastectomy } & 7(31.8) \\ \text { Lumpectomy } & 9(40.9) \\ \text { Treatment* } & \\ \text { Chemotherapy } & 10(45.5) \\ \text { Hormone therapy } & 14(63.6) \\ \text { Radiation therapy } & 14(63.6) \\ \text { Breast cancer recurrence } & \\ \text { None } & 20(83.3) \\ \text { One recurrence } & 1(4.2) \\ \text { Two recurrence } & 1(4.2) \\ \text { *almost all participants received a combination of treatments }\end{array}$

diurnal and reactive concentrations patterns. Bonferroni corrections were used to control for multiple comparisons. Violation of the assumption of sphericity, as indicated by a significant Mauchly's Test of Sphericity value, was offset by the Huynh-Feldt correction procedure, which adjusts the degrees of freedom [70].

\section{Diurnal secretory immunoglobulin A}

A $2 \times 5$ mixed design ANOVA was used to examine differences in mean SIgA concentrations collected at five time points over two consecutive days. The analyses were performed on the two-day average of the concentrations. The between-subject factor was group ( $B C$ survivors or 



Fig. 1. Mean diurnal SIgA concentrations over two consecutive days. Inset graphs include published diurnal cortisol and sAA for the same sample [21, 33]. Error bars represent standard error of the mean
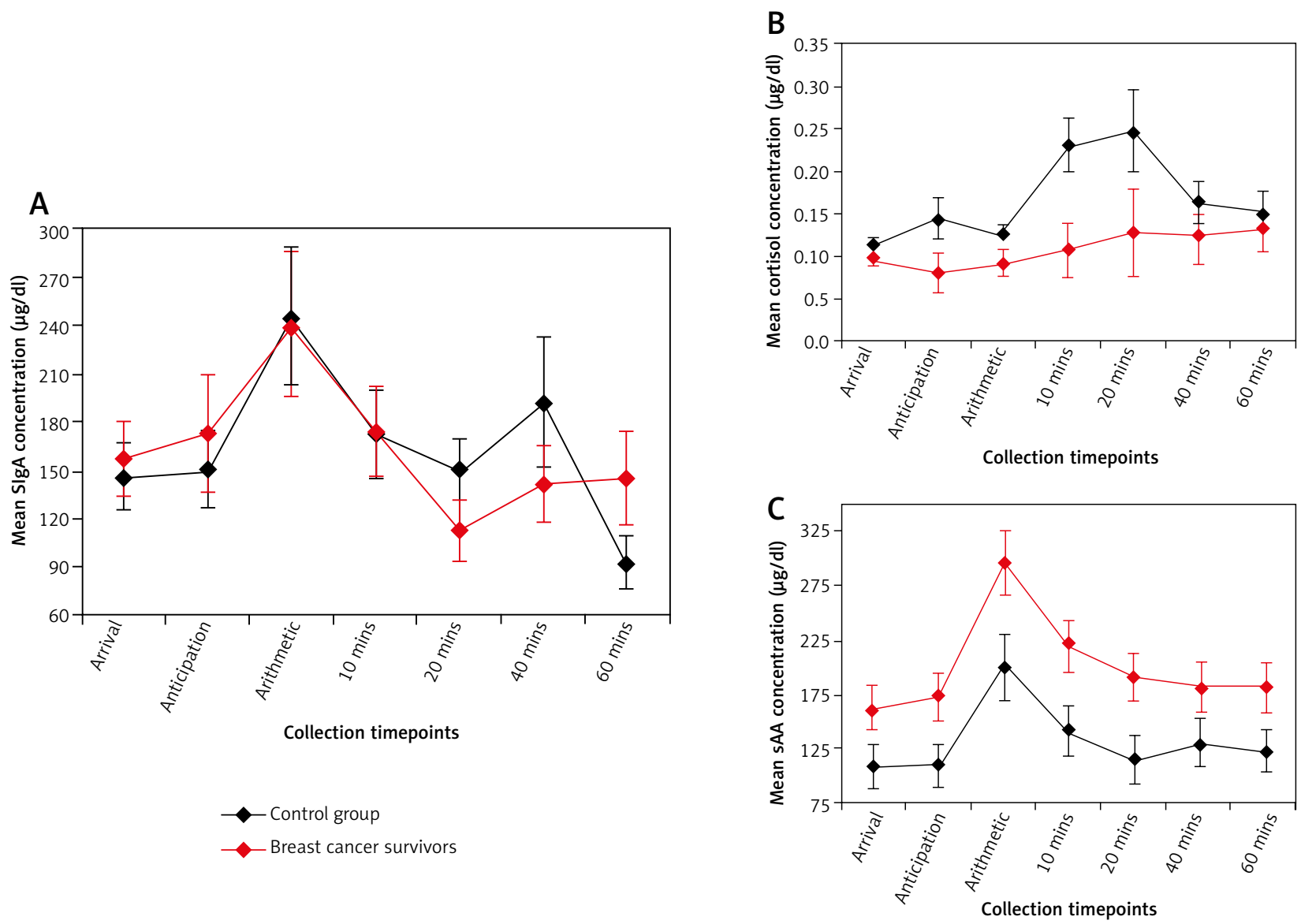

Fig. 2. Mean acute SlgA concentrations. Inset graphs include published diurnal cortisol and sAA for the same sample [21, 33]. Error bars represent standard error of the mean 
women with no history of $\mathrm{BC}$ ) and the within-subject factor was time (waking, 30 min after waking, at $1200 \mathrm{~h}$, $1600 \mathrm{~h}$, and $2100 \mathrm{~h}$ ).

Figure 1 A shows the plot of the diurnal SIgA concentrations. The inset graphs on the upper right side are the cortisol (Fig. 1B) and the SAA (Fig. 1C) data in the same subjects for comparison purposes [20,32]. Overall, both groups demonstrated similar diurnal SIgA patterns, with the highest concentration upon waking followed by a decrease to its nadir by $2100 \mathrm{~h}$. The statistical results revealed a significant main effect of time $\left(F(1.15,48.43)=60.40, p<0.001, \eta_{\mathrm{p}}{ }^{2}=0.59\right)$, no group effect $\left(F(1.42)=1.22, p=0.275, \eta_{p}^{2}=0.03\right)$, and a marginally significant group $\times$ time interaction $(F(1.15$, $\left.48.43)=3.71, p=0.054, \eta_{p}{ }^{2}=0.08\right)$. Overall, $B C$ survivors showed consistently elevated SAA values (Fig. 1C), but otherwise basal values of cortisol and SIgA were comparable to those of women with no history of cancer.

\section{Secretory immunoglobulin A in response to acute stress}

Figure 2A illustrates the profile of SIgA concentration patterns in response to an acute stressor, i.e. the TSST. The inset graphs represent the cortisol (Fig. 2B) and SAA (Fig. 2C) data for the same subjects for comparison purposes [20, 32]. A $2 \times 7$ mixed-design ANOVA was used to examine group and time differences in mean SIgA concentrations. The between-subject factor was group (BC survivors or healthy women), and the within-subject factor was time (arrival, anticipation, arithmetic, and $10 \mathrm{~min}, 20 \mathrm{~min}$, $40 \mathrm{~min}$, and $60 \mathrm{~min}$ after the TSST).

Both groups displayed a similar biphasic pattern with the first SIgA increase at arithmetic and the second increase at 40 minutes after the completion of the TSST. The analysis demonstrated a significant main effect of time $\left(F=8.60, p<0.001, \eta_{p}{ }^{2}=0.193\right)$ and no group $(F=0.001$, $\left.p=0.971, \eta_{p}{ }^{2}=0.000\right)$ or group $x$ time interaction $(F=1.65$, $\left.p=0.175, \eta_{p}^{2}=0.044\right)$. Across biomarkers, significant interactions were only found in cortisol concentrations in response to an acute stressor (Fig. 2B); the group differences observed in the SAA data (Fig. 2C) match their baseline rates (Fig. 1C) and therefore are not related to the stress exposure.

\section{Secretory immunoglobulin A reactivity profiles and medical characteristics}

Additional correlational analyses were performed in order to evaluate the relationship between BC characteristics such as the number of years since diagnosis, cancer stage, and type of treatment (chemotherapy or no chemotherapy), and the SIgA profile observed during the TSST. None of these analyses indicated any group differences based on medical variables. Furthermore, both groups showed the same degree of stress, as indicated by their VAS ratings, throughout the TSST [20].

\section{Discussion}

\section{Secretory immunoglobulin A findings}

The goal of the current study was to complement the findings on the concentration patterns of cortisol and SAA in BC survivors by exploring the diurnal and reactive profiles of an immune biomarker, i.e. SIgA, in the same participants. We reasoned that the HPA and SNS dysregulation found in BC survivors, as interpreted from cortisol and SAA patterns, respectively, might also be accompanied by abnormal SIgA concentrations, a biomarker of immunocompetence. However, our data showed no evidence of uncharacteristic SIgA basal or reactive secretion patterns, suggesting a normal and well-functioning immunological SIgA system.

Several studies have suggested an association between cancer experience and immune impairment [71-73], hence the reason why we selected SIgA as the candidate biomarker to evaluate immunocompetence in BC survivors. To our knowledge, only one study has measured salivary SIgA in women after the end of treatment for BC. Jensen et al. [73] surveyed a group of women diagnosed with $B C$ prior to treatment, during treatment, and six and 12 months after treatment. Their results indicated a significant decrease in SIgA in response to and following chemotherapy; the levels had normalised one year after treatment. They suggested that BC treatment depressed the SIgA producing plasma cells or inhibited the immunoglobulin transport mechanism in the salivary gland cells. As far as we know, the very few other studies that have used SIgA as a biomarker of immune function in a cancer population have investigated SIgA levels in relation to disease progression and/or prognosis and have not looked at BC specifically $[33,74,75]$.

Using biomarkers other than SIgA, other studies have also reported immune deficiency in cancer patients. For example, Campbell et al. [76] studied patients undergoing definitive surgery for BC and found a clear deficit in cytokine production, suggesting a general immune dysfunction in BC patients. They also noted that larger tumours were associated with more depressed levels of $T$ cell responses. A recent study by Verma et al. [71] found that antibody

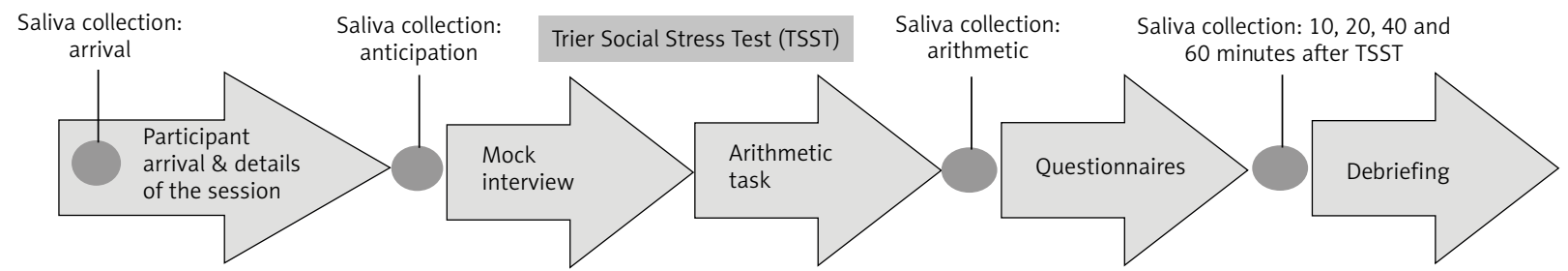

Fig. 3. Schematic representation of the laboratory session procedure 
levels never returned to their pre-cancer levels and that all types of lymphocytes significantly dropped after BC treatment. Similarly, Standish et al. [72] reported an important reduction in the numbers and functions of cells from both the adaptive and innate immune system following cancer treatment. Although these studies represent a significant contribution to the literature of cancer and immune impairment, none of them examined immunological dysfunction in long-term survivors of cancer (more than one year after treatment).

There are a few potential explanations for the fact that the SIgA concentration pattern of our BC survivor group did not differ from that of women with no history of cancer. First, low levels of SIgA are typically associated with the occurrence of different clinical diseases and infections [77-79]. Despite being survivors of BC, women in our study were perfectly healthy; they did not suffer from any post-cancer medical conditions (at least at the time of the saliva collection). Second, this similarity in the SIgA concentration patterns of our two groups may be due to recovery function of the immune system in $B C$ survivors [80]. Studies have shown that cancer patients typically exhibit functional immune response abnormalities up to 12 months after treatment, with the percentage of patients with entirely intact immune responses increasing gradually with time $[73,81]$. Because the saliva samples of our participants were collected on average 4.6 years after diagnosis, it is reasonable that the negative impact of the cancer experience on individuals' immunity may have normalised over time. Third, SIgA only makes up for a small proportion of all salivary immune ingredients and antibacterial proteins, including immunoglobulin and non-immunoglobulin [33]. Although salivary SIgA is defined as the primary means of assessing the body's "first line of defence" and is the most prolific antibody in saliva [33, 82], perhaps the measurement of other immunoglobulins such as immunoglobulin G or M or non-immunoglobulins such as lysozyme would have revealed more about the immune status of our participants.

\section{Cortisol, $\alpha$-amylase, and secretory immunoglobulin A findings}

Taken together, the SIgA findings reported here, complemented by the cortisol and SAA findings in the same individuals $[20,32]$ suggest three main conclusions. Breast cancer survivors, roughly five years after diagnosis: 1) exhibit a dysregulated HPA system, as indexed by a blunted cortisol pattern when confronted with an acute stressor, but otherwise had normal basal rhythms of the hormone; 2) displayed a dysregulated SNS on their elevated diurnal SAA concentration patterns, which was sustained in the face of an acute stressor; and 3) did not show mucosal immunity dysregulation, as demonstrated by their normal basal and reactive SIgA profiles.

Being diagnosed with cancer involves multiple complex and repeated stressful events over the course of diagnosis, treatment, and survivorship trajectory [83]. Our observation of a disruption of biological systems might, at least in part, be the result of these repeated "hits" of excessive stress that accompany a BC diagnosis and its aftermath [84]. Since the stress systems play a central role in the coordination and regulation of multiple system responses such as behavioural, physiological, and metabolic responses $[85,86]$, it is not surprising that HPA axis and SNS abnormalities have been associated with several negative outcomes such as depression, diabetes, post-traumatic stress disorder, metabolic dysfunction, hypertension, anxiety disorders, and cardiovascular diseases [87-90]. McGirr et al. [91] even found a relationship between dysregulated HPA axis and SNS and higher risk for suicide. Gaining a better understanding of how these abnormalities in stress systems lead to increased risks for various medical conditions is therefore primordial for the prevention and protection of vulnerable populations such as BC survivors.

The biological dysregulations found in our studies clearly indicate that BC survivors could benefit from additional follow-up care and post-treatment surveillance. Medical practices promoting regular and consistent monitoring for the late effects of cancer by physicians should be implemented in cancer care routine. The elaboration of health promotion and lifestyle intervention strategies aiming to optimise health after cancer should be a basic consideration and achieved based on the recommendations of the many studies that have identified factors associated with psychological and physical health in cancer survivors. For instance, interventions promoting social support, purpose in life, self-acceptance, and expressive activities have been shown to maximise survivors' health outcomes [92, 93]. Indeed, many studies have suggested that psychological interventions following cancer treatment reduce the stress associated with the cancer survivorship trajectory, decrease the risk of recurrence, and enhance positive coping, quality of life, and physiological responses, such as those studied in the current study $[12,94,95]$. It would therefore be beneficial to consider the incorporation of such services into standard medical care for cancer survivors.

With the number of cancer survivors climbing each year, efforts to better understand, monitor, and mitigate the physiological consequences of a cancer experience is critical. This study is the first to describe long-term trajectories of cancer-related stress in BC survivors by examining SIgA levels specifically and comparing them to cortisol and SAA levels in the same women. The present work allows us to further elucidate the physiological sequelae of a cancer experience on the human stress systems and has important clinical significance as it represents a step forward towards the development of specific guidelines and practical recommendations for optimal cancer survivorship care plan. Moreover, as some studies found that being a survivor of cancer may divert attention away from other health conditions, thereby leading to delayed healthcare [96], it is even more important to educate people and raise awareness in public health organisations about the adverse long-term effects of a cancer experience.

\section{Limitations and future directions}

First, the sample size in our study is small, albeit comparable to that observed in other published reports of 
this nature [72, 75, 97]; these study protocols are quite demanding, making recruitment very challenging. Second, while we did compare our clinical results to that of a disease-free sample, it would have been useful to have had information on the baseline status of the biomarkers we examined in our studies. Third, it would be ideal to include the measurement of other immunoglobulin levels in order to allow for a broader assessment of BC survivors' immune functioning. Because of: a) SIgA underinvestigation in the $B C$ survivor population, b) its critical role in immune function, and $c$ ) its direct impact on many anti-inflammatory and immunological responses, its assessment appeared to be the most pertinent biomarker of stress and immunity to examination in this study, given our limited resources. Finally, the reliance on convenience sampling may undermine the ability to extend our findings to the BC survivor population as a whole.

This study prompts several future research directions and objectives. With all the notable evidence derived from cancer survivorship research on the long-term adverse effects of a cancer experience, it is essential now that cancer research not solely focus on curing the underlying disease but also simultaneously strive towards finding interventions with minimal toxicity but optimal effectiveness, in order to limit the undesirable post-treatment outcomes. Future studies should not only investigate biomarkers and their underlying mechanisms but should also explore ways and methods of reversing these biological dysfunctions caused by the cancer experience. Furthermore, because most of the cancer survivorship research to date has employed cross-sectional cohorts, longitudinal studies examining the trajectories of the physiological and biological consequences of the cancer experience over time are essential to evaluate decrements in survivor functioning. Finally, it is crucial for future cancer survivorship studies to consider including immune biomarkers such as SIgA more often in their design because components of the innate mucosal system are vital in the body's defence against pathogens.

\section{Conclusions}

There are still many gaps in the literature that need to be addressed in order to gain a better overall understanding of the consequences of a BC experience on an individual's stress-related physiological functioning. Our results indicate certain abnormalities in biological systems following a cancer experience and emphasise the need for continued research in this ever-growing cancer survivor population. They further suggest that our ongoing healthcare and support services be re-evaluated in order to ameliorate the late and long-term effects of a cancer experience. Since the population of cancer survivors continues to grow each year, cancer survivorship needs to be a research priority in order to keep up with their needs.

\section{Acknowledgements}

We gratefully acknowledge the women who participated in this study, without whom our research could not be conducted. We also want to thank the Canadian Breast Cancer Research Alliance for their generous funding of our work.

\section{The authors declare no conflict of interest.}

\section{References}

1. Canadian Cancer Society Breast Cancer in Canada. http://www. cbcf.org/ontario/AboutBreastCancerMain/FactsStats/Pages/ Breast-Cancer-Canada.aspx.

2. Knobf MT. Psychosocial responses in breast cancer survivors. Semin Oncol Nurs 2007; 23: 71-83.

3. Harrington CB, Hansen JA, Moskowitz M, Todd BL, Feuerstein M. It's not over when it's over: long-term symptoms in cancer survivors - a systematic review. Int J Psychiatry Med 2010; 40: 163-181.

4. Shi O, Smith TG, Michonski JD, Stein KD, Kaw C, Cleeland CS. Symptom burden in cancer survivors 1 year after diagnosis. Cancer 2011; 117: 2779-2790.

5. Green McDonald P, O'Connell M, Lutgendorf SK. Psychoneuroimmunology and cancer: a decade of discovery, paradigm shifts, and methodological innovations. Brain Behav Immun 2013; 30 (Suppl): S1-9.

6 Dhabhar FS. Enhancing versus Suppressive Effects of Stress on Immune Function: Implications for Immunoprotection and Immunopathology. Neuroimmunomodulation 2009; 16: 300-317.

7. Huss R. Biomarkers. In: Translational Regenerative Medicine. Academic Press, Boston 2015: 235-241.

8. Abercrombie HC, Giese-Davis J, Sephton S, Epel ES, Turner-Cobb JM, Spiegel D. Flattened cortisol rhythms in metastatic breast cancer patients. Psychoneuroendocrinology 2004; 29: 1082-1092.

9. Sephton SE, Sapolsky RM, Kraemer HC, Spiegel D. Diurnal cortisol rhythm as a predictor of breast cancer survival. J Natl Cancer Inst 2000; 92: 994-1000

10. Fulford AJ, Harbuz MS. An introduction to the HPA axis. In: Handbook of Stress and the Brain. Elsevier Science Ltd, Amsterdam 2005.

11. Kirschbaum C, Hellhammer DH. Encyclopedia of Stress: Salivary cortisol. Academic Press, San Diego 2000.

12. McGregor BA, Antoni MH. Psychological intervention and health outcomes among women treated for breast cancer: a review of stress pathways and biological mediators. Brain Behav Immun 2009; 23: 159-166.

13. Armario A, Vallès A, Dal-Zotto S, Márquez C, Belda X. A single exposure to severe stressors causes long-term desensitisation of the physiological response to the homotypic stressor. Stress 2004; 7 : 157-172.

14. Parker KJ, Schatzberg AF, Lyons DM. Neuroendocrine aspects of hypercortisolism in major depression. Horm Behav 2003; 43: 60-66.

15. Peeke PM, Chrousos GP. Hypercortisolism and obesity. Ann N Y Acad Sci 1995; 771: 665-676.

16. Chiodini I, Torlontano M, Scillitani A, et al. Association of subclinical hypercortisolism with type 2 diabetes mellitus: a case-control study in hospitalized patients. Eur J Endocrinol 2005; 153: 837-844.

17. Sapolsky RM, Alberts SC, Altmann J. Hypercortisolism associated with social subordinance or social isolation among wild baboons. Arch Gen Psychiatry 1997; 54: 1137-1143.

18. Fries E, Hesse J, Hellhammer J, Hellhammer DH. A new view on hypocortisolism. Psychoneuroendocrinology 2005; 30: 1010-1016.

19. Heim C, Ehlert U, Hellhammer DH. The potential role of hypocortisolism in the pathophysiology of stress-related bodily disorders. Psychoneuroendocrinology 2000; 25: 1-35.

20 Couture-Lalande, M-Ė, Lebel S, Bielajew C. Analysis of the cortisol diurnal rhythmicity and cortisol reactivity in long-term breast cancer survivors. Breast Cancer Manag 2014; 3: 465-476.

21. Porter LS, Mishel M, Neelon V, Belyea M, Pisano E, Soo MS. Cortisol levels and responses to mammography screening in breast cancer survivors: a pilot study. Psychosom Med 2003; 65: 842-848. 
22. Spiegel D, Giese-Davis J, Taylor CB, Kraemer H. Stress Sensitivity in Metastatic Breast Cancer: Analysis of Hypothalamic-Pituitary-Adrenal Axis Function. Psychoneuroendocrinology 2006; 31: 1231-1244.

23. Nater $U$, Rohleder $N$. Salivary alpha-amylase as a non-invasive biomarker for the sympathetic nervous system: current state of research. Psychoneuroendocrinology 2009; 34: 486-496.

24. Rohleder N, Nater U, Wolf JM, Ehlert U, Kirschbaum C. Psychosocial stress-induced activation of salivary alpha-amylase: an indicator of sympathetic activity? Ann N Y Acad Sci 2004; 1032: 258-263.

25. Yong HW, Zailina H, Zubaidah JO, Saidi M, Yong HY, Zalilah MS. The relationship between psychological distress with salivary $\alpha$-amylase and stress related symptoms among breast cancer survivors. Health 2013; 5: 811.

26. Nater UM, Rohleder N, Schlotz W, Ehlert U, Kirschbaum C. Determinants of the diurnal course of salivary alpha-amylase. Psychoneuroendocrinology 2007; 32: 392-401.

27. Bosch JA, Brand H, Ligtenberg A, Bermond B, Hoogstraten J, Nieuw Amerongen, AV. The response of salivary protein levels and SIgA to an academic examination are associated with daily stress. J Psychophysiol 1998; 12: 384-391.

28. Chatterton RT, Vogelsong KM, Lu YC, Hudgens GA. Hormonal responses to psychological stress in men preparing for skydiving. Clin Endocrinol Metab 1997; 82: 2503-2509.

29. Behringer V, Deschner T, Möstl E, Selzer D, Hohmann G. Stress af fects salivary alpha-Amylase activity in bonobos. Physiol Behav 2012; 105: 476-482.

30. Almela M, Hidalgo V, Villada C, van der Meij L, Espín L, Gómez-Amor J, Salvador A. Salivary alpha-amylase response to acute psychosocial stress: the impact of age. Biol Psychol 2011; 87: 421-429.

31. Liu H, Dong W, Wang J, Wang T, Hu P, Wei S, Ye L, Wang Q. Association between salivary $\alpha$-amylase activity and pain relief scale scores in cancer patients with bone metastases treated with radiotherapy. Chin Med J 2013; 126: 4444-4447.

32. Wan C, Couture-Lalande M-Ė, Narain TA, Lebel S, Bielajew C. Salivary Alpha-Amylase Reactivity in Breast Cancer Survivors. Int J Environ Res Public Health 2016; 13: 353.

33. Sun H, Chen Y, Zou X, et al. Salivary Secretory Immunoglobulin (SIgA) and Lysozyme in Malignant Tumor Patients. Biomed Res Int 2016, 2016: 8701423

34. Bellussi L, Cambi J, Passali D. Functional maturation of nasal mu cosa: role of secretory immunoglobulin A (SIgA). Multidiscip Respir Med 2013; 8: 46

35. Widmaier EP, Raff H, Strang KT. Vander's Human Physiology the Mechanisms of Body Function. 12 ${ }^{\text {th }}$ ed. McGraw-Hill, New York, 2011.

36. Bosch JA, Ring C, de Geus EJC, Veerman ECI, Amerongen AVN. Stress and secretory immunity. Int Rev Neurobiol 2002; 52: 213-253.

37. Moreira A, de Moura NR, Coutts A, Costa EC, Kempton T, Aoki MS. Monitoring internal training load and mucosal immune responses in futsal athletes. J Strength Cond Res 2013; 27: 1253-1259.

38. Hucklebridge F, Clow A, Evans P. The relationship between salivary secretory immunoglobulin $A$ and cortisol: neuroendocrine response to awakening and the diurnal cycle. Int J Psychophysio 1998; 31: 69-76.

39. Li T-L, Gleeson M. The effect of single and repeated bouts of pro longed cycling and circadian variation on saliva flow rate, immunoglobulin A and alpha-amylase responses. J Sports Sci 2004; 22: 1015-1024.

40. Rantonen PJ, Meurman JH. Correlations Between Total Protein, Lysozyme, Immunoglobulins, Amylase, and Albumin in Stimulated Whole Saliva During Daytime. Acta Odontol Scand 2000; 58: 160-165.

41. Teeuw W, Bosch JA, Veerman ECI, Amerongen AVN. Neuroendocrine regulation of salivary IgA synthesis and secretion: implications for oral health. Biol Chem 2004; 385: 1137-1146.

42. Tsujita S, Morimoto K. Secretory IgA in saliva can be a useful stress marker. Environ Health Prev Med 1999; 4: 1-8.

43. Valdimarsdottir HB, Stone AA. Psychosocial factors and secretory immunoglobulin A. Crit Rev Oral Biol Med 1997; 8: 461-474.

44. Müller F, Holberg-Petersen M, Rollag H, Degré M, Brandtzaeg P, Frøland SS. Nonspecific oral immunity in individuals with HIV infection. J Acquir Immune Defic Syndr 1992; 5: 46-51.
45. Brown AM, Lally ET, Frankel A, Harwick R, Davis LW, Rominger CJ. The association of the IGA levels of serum and whole saliva with the progression of oral cancer. Cancer 1975; 35: 1154-1162.

46. Cohen S, Williamson GM. Stress and infectious disease in humans. Psychol Bull 1991; 109: 5-24.

47. Jemmott JB, Locke SE. Psychosocial factors, immunologic mediation, and human susceptibility to infectious diseases: how much do we know? Psychol Bull 1984; 95: 78-108.

48. Kiecolt-Glaser JK, Garner W, Speicher C, Penn GM, Holliday J, Glaser R. Psychosocial modifiers of immunocompetence in medical students. Psychosom Med 1984; 46: 7-14.

49. Segerstrom SC, Miller GE. Psychological stress and the human immune system: a meta-analytic study of 30 years of inquiry. Psychol Bull 2004; 130: 601-630.

50. Evans P, Clow A, Hucklebridge F. Stress and the immune system: Current issues and directions in research. Psychology 1997; 10: 303-307.

51. Vermeer HJ, van Ijzendoorn MH, Groeneveld MG, Granger DA. Downregulation of the immune system in low-quality child care: the case of secretory immunoglobulin A (SIgA) in toddlers. Physiol Behav 2012; 105: 161-167.

52. Kvietkauskaite R, Vaicaitiene R, Mauricas $M$. The change in the amount of immunoglobulins as a response to stress experienced by soldiers on a peacekeeping mission. Int Arch Occup Environ Health 2014; 87: 615-622.

53. Yang Y, Koh D, Ng V, et al. Self perceived work related stress and the relation with salivary IgA and lysozyme among emergency department nurses. Occup Environ Med 2002; 59: 836-841.

54. Kugler J, Reintjes F, Tewes V, Schedlowski M. Competition stress in soccer coaches increases salivary. Immunoglobin A and salivary cortisol concentrations. J Sports Med Phys Fitness 1996; 36: 117 120

55. Groer M, Murphy R, Bunnell W, Salomon K, Van Eepoel J, Rankin B, White K, Bykowski C. Salivary measures of stress and immunity in police officers engaged in simulated critical incident scenarios. J Occup Environ Med 2010; 52: 595-602.

56. Campisi J, Bravo Y, Cole J, Gobeil K. Acute psychosocial stress differentially influences salivary endocrine and immune measures in undergraduate students. Physiol Behav 2012; 107: 317-321.

57. Wolf JM, Nicholls E, Chen E. Chronic stress, salivary cortisol, and alpha-amylase in children with asthma and healthy children. Biol Psychol 2008; 78: 20-28.

58. Koh DS, Koh GC. The use of salivary biomarkers in occupational and environmental medicine. Occup Environ Med 2007; 64: 202 210

59. Salimetrics Salivary Secretory IgA Enzyme Immunoassay Kit. In: Salimetrics. Carlsbad, CA, USA 2015.

60. Hellhammer J, Schubert M. The physiological response to Trier Social Stress Test relates to subjective measures of stress during but not before or after the test. Psychoneuroendocrinology 2012; 37: 119-124.

61. Allen AP, Kennedy PJ, Cryan JF, Dinan TG, Clarke G. Biological and psychological markers of stress in humans: focus on the Trier Social Stress Test. Neurosci Biobehav Rev 2014; 38: 94-124.

62 Trueba AF, Mizrachi D, Auchus RJ, Vogel PD, Ritz T. Effects of psychosocial stress on the pattern of salivary protein release. Physiol Behav 2012; 105: 841-849.

63. Kudielka B, Hellhammer DH, Kirschbaum C. Ten years of research with the Trier Social Stress Test (TSST) - revisited. In: Fundamentals in social neuroscience. Guilford Press, New York 2007.

64. Kirschbaum C, Pirke KM, Hellhammer DH. The "Trier Social Stress Test” - a tool for investigating psychobiological stress responses in a laboratory setting. Neuropsychobiology 1993; 28: 76-81.

65. Aitken RC. Measurement of feelings using visual analogue scales. Proc R Soc Med 1969; 62: 989-993.

66. Brantley PJ, Waggoner CD, Jones GN, Rappaport NB. A Daily Stress Inventory: development, reliability, and validity. J Behav Med 1987; 10: 61-74.

67. Cohen S, Kamarck T, Mermelstein R. A global measure of perceived stress. J Health Soc Behav 1983; 24: 385-396. 
68. Sarason IG, Johnson JH, Siegel JM. Assessing the impact of life changes: development of the Life Experiences Survey. J Consult Clin Psychol 1978; 46: 932-946.

69. Henson BS, Wong DT. Collection, Storage, and Processing of Saliva Samples for Downstream Molecular Applications. In Oral Biology. Methods in Molecular Biology. Humana Press, Totowa, NJ 2010, Vol. 666: 21-30.

70. Tabachnick BG, Fidell LS. Using multivariate statistics; $6^{\text {th }}$ ed. Pearson Education. Upper Saddle River, NJ, USA 2013.

71. Verma R, Foster RE, Horgan K, Mounsey K, Nixon H, Smalle N, Hughes TA, Carter CRD. Lymphocyte depletion and repopulation after chemotherapy for primary breast cancer. Breast Cancer Res 2016; 18: 10.

72. Standish LJ, Torkelson C, Hamill FA, et al. Immune defects in breast cancer patients after radiotherapy. I Soc Integr Oncol 2008; 6: 110-121.

73. Jensen SB, Mouridsen HT, Reibel J, Brünner N, Nauntofte B. Adjuvant chemotherapy in breast cancer patients induces temporary salivary gland hypofunction. Oral Oncol 2008; 44: 162-173.

74. Rizvi S, Yang JD, Gores GJ. Anti-GP2 IgA: a biomarker for disease severity and/or cholangiocarcinoma in primary sclerosing cholangitis? Gut 2017; 66: 4-5

75. Zhang S, Zhang X, Yin K, Li T, Bao Y, Chen Z. Variation and significance of secretory immunoglobulin A, interleukin 6 and dendritic cells in oral cancer. Oncol Lett 2017; 13: 2297-2303.

76. Campbell MJ, Scott J, Maecker HT, Park JW, Esserman LJ. Immune dysfunction and micrometastases in women with breast cancer. Breast Cancer Res Treat 2005; 91: 163-171.

77. Vo Ngoc L, Krist L, van Overveld FJ, Rijkers GT. The long and winding road to IgA deficiency: causes and consequences. Expert Rev Clin Immunol 2017; 13: 371-382.

78. Yazdani R, Latif A, Tabassomi F, Abolhassani H, Azizi G, Rezaei N, Aghamohammadi A. Clinical phenotype classification for selective immunoglobulin A deficiency. Expert Rev Clin Immunol 2015; 11: 1245-1254.

79. Ludvigsson JF, Neovius M, Hammarström L. Risk of Infections Among 2100 Individuals with IgA Deficiency: a Nationwide Cohort Study. J Clin Immunol 2016; 36: 134-140.

80. Thornton LM, Andersen BL, Crespin TR, Carson WE. Individual trajectories in stress covary with immunity during recovery from cancer diagnosis and treatments. Brain Behav Immun 2007; 21: 185-194.

81. Kang D-H, Weaver MT, Park N-J, Smith B, McArdle T, Carpenter J. Significant Impairment in Immune Recovery Following Cancer Treatment. Nurs Res 2009: 58; 105-114.

82. Holmgren J, Czerkinsky C. Mucosal immunity and vaccines. Nat Med 2005; 11: S45-53

83. Swartzman S, Booth JN, Munro A, Sani F. Posttraumatic stress disorder after cancer diagnosis in adults: A meta-analysis. Depress Anxiety 2017; 34: 327-339.

84. Saligan LN, Olson K, Filler K, et al. The biology of cancer-related fatigue: a review of the literature. Support Care Cancer 2015; 23 : 2461-2478.

85. McEwen BS. Stress, adaptation, and disease. Allostasis and allostatic load. Ann N Y Acad Sci 1998; 840: 33-44.

86. Kinlein SA, Wilson CD, Karatsoreos IN. Dysregulated Hypothalamic-Pituitary-Adrenal Axis Function Contributes to Altered Endocrine and Neurobehavioral Responses to Acute Stress. Front Psychiatry 2015; 6: 31.

87. de Kloet CS, Vermetten E, Geuze E, Kavelaars A, Heijnen CJ, Westenberg HG. Assessment of HPA-axis function in posttraumatic stress disorder: pharmacological and non-pharmacological challenge tests, a review. J Psychiatr Res 2006; 40: 550-567.

88. Pariante CM, Lightman SL. The HPA axis in major depression: clas sical theories and new developments. Trends Neurosci 2008; 31 464-468.

89. Rosmond R. Stress induced disturbances of the HPA axis: a pathway to Type 2 diabetes? Med Sci Monit 2003; 9: 35-39.
90. Kumari M, Shipley M, Stafford M, Kivimaki M. Association of diurnal patterns in salivary cortisol with all-cause and cardiovascular mortality: findings from the Whitehall II study. J Clin Endocrinol Metab 2011; 96: 1478-1485.

91. McGirr A, Diaconu G, Berlim MT, Pruessner JC, Sablé R, Cabot S, Turecki G. Dysregulation of the sympathetic nervous system, hypothalamic-pituitary-adrenal axis and executive function in individuals at risk for suicide. J Psychiatry Neurosci 2010; 35: 399-408.

92. Andrykowski MA, Lykins E, Floyd A. Psychological health in cancer survivors. Semin Oncol Nurs 2008; 24: 193-201.

93. Falagas ME, Zarkadoulia EA, Ioannidou EN, Peppas G, Christodoulou C, Rafailidis PI. The effect of psychosocial factors on breast cancer outcome: a systematic review. Breast Cancer Res 2007; 9: R44.

94. Faller H, Schuler M, Richard M, Heckl U, Weis J, Küffner R. Effects of Psycho-Oncologic Interventions on Emotional Distress and Quality of Life in Adult Patients With Cancer: Systematic Review and Meta-Analysis. J Clin Oncol 2013; 31: 782-793.

95. Andersen BL, Goyal NG, Westbrook TD, Bishop B, Carson WE. Trajectories of Stress, Depressive Symptoms, and Immunity in Cancer Survivors: Diagnosis to 5 Years. Clin Cancer Res 2017; 23: 52-61.

96. Earle CC, Neville BA. Under use of necessary care among cancer survivors. Cancer 2004; 101: 1712-1719.

97. Tsavaris N, Kosmas C, Vadiaka M, Kanelopoulos P, Boulamatsis D. Immune changes in patients with advanced breast cancer undergoing chemotherapy with taxanes. Br J Cancer 2002; 87: 21-27.

\section{Address for correspondence}

\section{Maude Lambert}

University of Ottawa

136 Jean-Jacques Lussier, Vanier Hall

K1N 6N5 Ottawa, Canada

e-mail:mlamb079@uottawa.ca

Submitted: 21.06 .2018

Accepted: 13.09 .2018 